\title{
Our Study is Just an Introduction, Reply to: Noncompaction in Healthy Subjects, Dilated and Hypertrophic Cardiomyopathy, and Neuromuscular Disorders Is the Same Entity
}

\author{
Keyhan Sayadpour Zanjani, and Ahmad Gamei Khosroshahi ${ }^{2}$ \\ ${ }^{1}$ Division of Pediatrics, Children's Medical Center, Tehran University of Medical Sciences, Tehran, IR Iran \\ ${ }^{2}$ Division of Pediatrics, Shahid Madani Hospital, Tabriz University of Medical Sciences, Tabriz, IR Iran \\ ${ }^{*}$ Corresponding author: Keyhan Sayadpour Zanjani, Division of Pediatrics, Children's Medical Center, Tehran University of Medical Sciences, Tehran, IR Iran. Fax: +98-2122132219, \\ E-mail: ksayadpour@yahoo.com \\ Received: June 22, 2015; Accepted: July 1, 2015
}

Keywords: Left Ventricular Noncompaction 1; Diagnosis; Magnetic Resonance Imaging

\section{Dear Editor,}

The interest of one of the most famous investigators in the world about left ventricular noncompaction (LVNC) in our article $(1,2)$ is a great honor and very much appreciated. In that article, we proposed a new method for echocardiographic diagnosis of LVNC. This method is in fact based on the magnetic resonance imaging (MRI) diagnosis of LVNC which measures the percentage of noncompacted myocardium to the whole left ventricular muscle mass (3).

Our article is just introduction of a new method. We believe that it should be evaluated on more cases, not only in children but also in adults. We work in a pediatric center with no adult patients and limited number of LVNC cases. Although we extended the time period, we collected only 4 cases of LVNC meanwhile. This number is too low for assessing any probable effect of sex, age, or ethnicity. The threshold value of $17 \%$ was based on this number of patients. This needs to be reevaluated on a larger cohort of patients to reach a reliable value with acceptable sensitivity and specificity. However, it is very near to the MRI threshold of $20 \%$, could diagnose all 4 cases of LVNC and rule out all 26 cases of dilated cardiomyopathy except 2 (less than $8 \%$ of false positive cases).

All measurements and echocardiographic examinations were performed by one coauthor and no interobserver variability was evaluated. Limited intraobserver variability was controlled at the beginning of the study, yielding acceptable results. The echocardiographer used his maximal effort not including papillary muscles, bands, and chordae tendineae in the noncompacted area. However, sometimes it was very hard to distinguish them.

Unfortunately, the image in the article is not of sufficient clarity to show all details. Using an echocardiogram with acceptable 2-dimensional resolution, it is possible to distinguish ventricular cavity, compacted and noncompacted myocardium. In some patients, transthoracic echocardiogram yields suboptimal images. We excluded some patients with suboptimal echocardiogram from the study.

Although LVNC is most pronounced in the apical area of $\mathrm{LV}$, it can be found in the other areas as well. Thinner myocardium of the cardiac base made the percentage at this level not much lower than in the cardiac apex.

Echocardiographies were done using a 4 - $8 \mathrm{~Hz}$ P10 transducer from SonoSite MicoMaxx®. We tried to capture true short axis views perpendicular to the cardiac axis. However, in some patients we angulated our transducer to obtain a clear view for the measurements. Pulmonary hyperaeration in the patients with decreased cardiac function was a contributing factor.

We had no intraoperative biopsy or postmortem autopsy examination. All of our patients were diagnosed after the neonatal period and had no prior normal echocardiographic examination. We have not noted endocardial fibrosis in any of our patients. Neurologic examination was not part of our study, but we will examine our patients for neuromuscular disease regarding its high prevalence in LVNC patients (4).

We have only compared our criteria with that of Jenni et al. (5). It is advisable to compare it with the other echocardiographic criteria and especially with MRI findings. Unfortunately, cardiac MRI for children was not available to us when launching this study.

Lastly, we tried to introduce a new echocardiographic method for the diagnosis of LVNC based on the known MRI criteria. We recognized our limitations in the num-

Copyright (C) 2015, Growth \& Development Research Center. This is an open-access article distributed under the terms of the Creative Commons Attribution-NonCommercial 4.0 International License (http://creativecommons.org/licenses/by-nc/4.0/) which permits copy and redistribute the material just in noncommercial usages, provided the original work is properly cited. 
ber of patients and quality of the echocardiograms. However, if the authors or other investigators interested in this disease accept the logical basis of our proposed criteria, we invite them to use, refine, and investigate it on their patients.

\section{References}

1. Khosroshahi AG, Zanjani KS, Kocharian A, Zeinaloo A, Kiani A, Rad EM. A novel method for quantification of left ventricular noncompaction using two-dimensional echocardiography in children. Iran J Pediatr. 2013;23(5):519-24.
2. Stollberger C, Finsterer J. Noncompaction in healthy subjects, dilated and hypertrophic cardiomyopathy, and neuromascular disorders is the same entity. Iran J Pediatr. 2015;25(2):1-2.

3. Korcyk D, Edwards CC, Armstrong G, Christiansen JP, Howitt L, Sinclair T, et al. Contrast-enhanced cardiac magnetic resonance in a patient with familial isolated ventricular non-compaction. $J$ Cardiovasc Magn Reson. 2004;6(2):569-76.

4. Finsterer J, Stollberger C, Schubert B. Acquired left ventricular noncompaction as a cardiac manifestation of neuromuscular disorders. Scand Cardiovasc J. 2008;42(1):25-30.

5. Jenni R, Oechslin E, Schneider J, Attenhofer Jost C, Kaufmann PA. Echocardiographic and pathoanatomical characteristics of isolated left ventricular non-compaction: a step towards classification as a distinct cardiomyopathy. Heart. 2001;86(6):666-71. 